\title{
The electronic properties of Aurum-doped with both-edge narrow armchair graphene nanoribbons
}

\author{
Weihua Wang ${ }^{1, \text { a }}$, Cuilan Zhao ${ }^{1, \mathrm{~b}}$, Peifang $\mathrm{Li}^{1, \mathrm{c}}$ \\ ${ }^{1}$ College of Physics and Electronic Information, Inner Mongolia University for Nationalities, \\ Tongliao 028000, China \\ a chenqiwangweihua@163.com \\ bnmdzcl@163.com \\ c13614419644@163.com
}

Keywords: Both-edge Au-doped armchair graphene nanoribbons; charge density; electronic energy band structure; project density of states

\begin{abstract}
The charge density, electronic energy band structure and density of states of Au-doped armchair graphene nanoribbons (AGNRs) with both-edge are investigated using the local density approximation based on density function theory. Our results indicate the charge density is transferred and mainly located on the Au atoms. The formation energy of Au-doped AGNRs is calculated for one-edge, both-edge and centre. So we predict that one-edge Au-doped AGNRs is an energetically favorable practice. The energy band structure shows extra bands at near Fermi level in the valence band, which is cause to promote conductivity. The project density of states is calculated and reveals that the localization and hybridization between $\mathrm{C}-2 p$, Au- $6 s, 6 p, 5 d$ and $\mathrm{H}-1$ s electronic states are much stronger in the valence band and the conduction band group. A localization state is induced due to the absence of the bonding charge between $\mathrm{Au}$ and $\mathrm{H}$ atoms, which contributes to $\mathrm{H}-1 \mathrm{~s}$ electronic states at $-0.21 \mathrm{eV}$ near the Fermi level. It causes Fermi level is crossed by the conduction band to make becoming metallic.
\end{abstract}

\section{Introduction}

Graphene nanoribbons (GNRs) of various widths have been synthesized using the mechanical method and the epitaxial growth method ${ }^{[1]}$. The electronic properties of GNRs are determined by the shape of the edges and incorporation of impurities ${ }^{[2,3]}$. The GNRs are suited to doping, due to the active sites of the dangling bonds ${ }^{[4]}$. The stability and electronic properties of AGNRs with doping Mg, B, S, Ti and Mn atoms are investigated using density functional theory by S.S Chauhan et $\mathrm{al}^{[5]}$. They predict that transition metals as substitutional impurities in AGNRs are energetically more favorable and minimize the band gap. However, the models of these investigations are not obtained by experiment. Xiaowei Zhang et $\mathrm{al}^{[6]}$ investigated GNRs were obtained by hydrogen plasma treatment of chemically unzipped carbon nanotubes deposited onto an Au (111) substrate. So Au doped the AGNRs is investigated to obtain electronic properties with $\mathrm{H}$ terminated AGNRs edges. The Au-doped AGNRs for different configurations have not been previously studied. In this paper, we investigate the charge density, electronic energy band and density of states of Au-doped narrow $\mathrm{H}$-terminated AGNRs for both-edge doping configurations, using first principle calculations based on density functional theory.

\section{Modeling and theoretical Methods}

The charge density, electronic energy band and density of states of both-edge Au-doped H-terminated narrow AGNRs are studied using the local density approximation (LDA) based on density function theory (DFT). The LDA with exchange-correlation function and norm-conserving pseudopotential terms was used. The plane-wave cutoff energy was set to $125 \mathrm{Ry}$. The numerical integration of the Brillouin zone was performed using a k-mesh of dimensions $\mathbf{1} \times \mathbf{1} \times \mathbf{4 1}$. The AGNRs were modeled in a supercell along the $z$-direction ${ }^{[10]}$. The modeling was done 
with XCrySDen ${ }^{[7]}$. The width of the AGNRs represents the number of C-C pairs forming the width of the ribbon. The $\mathrm{C}-\mathrm{C}$ bond length is $1.42 \AA$ for experiment value of bare AGNRs. We model Au-doped AGNRs, with Au-doping at different configurations for both-edge, by substituting $\mathrm{C}$ atoms in the supercell by Au atoms, as shown in Figure 1. All of the internal structural parameters of the doped AGNRs were optimized by minimizing the total energy and the quasi-Newtonian algorithm. The electron occupation number was determined by the Methfessel-Paxton (M-P) method $^{[8]}$. The electronic states C-2s, $2 p$, Au-6s, $6 p, 5 d$ and $\mathrm{H}-1 s$ are considered in our calculations. The DFT calculations were performed using the QUANTUM ESPRESSO package ${ }^{[9]}$.
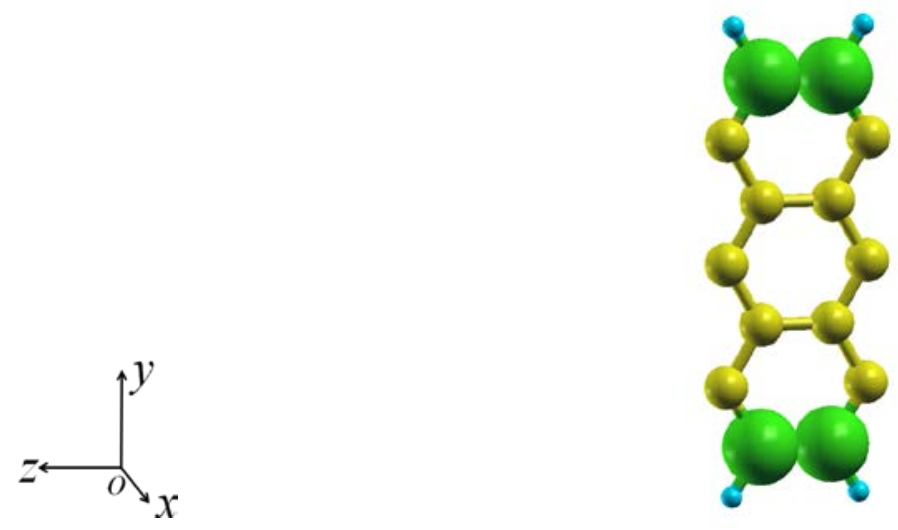

Fig.1. Schematic diagram of both-edge Au-doped AGNRs.

\section{Results and discussion}

\section{Stability and analysis}

To analyze the stability of Au-doping AGNRs with one-edge, both-edge and centre configurations of nanoribbons, we calculated the formation energy for different configurations, as shown Table 1 . The formation energy can be written as [9]

$$
E_{F}=E_{T}+n E(C)-E_{H}-m E(A u)
$$

where $E_{T}$ represents the total energy of Au-doped $H$ terminated AGNRs with doping one-edge configurations; $E_{H}$ is the total energy of $H$ terminated AGNRs; and $E(C)$ and $E(A u)$ represent the total energy of an isolated $\mathrm{C}$ and $\mathrm{Au}$ atom, respectively. The formation energy of one-edge Au-doped AGNRs are the lowest. It can be predicted that one-edge Au-doped AGNRs is more close to the practice. It is stability for Au-doping AGNRs with one-edge. The both-edge Au-doping is not so stable due to higher the formation energy.

Table 1 The calculated formation energy (eV) of Au-doped AGNRs with different configurations.

\begin{tabular}{cc}
\hline Configurations & Formation energy (eV) \\
\hline One-edge Au-doped & 11.68 \\
Centre Au-doped & 14.63 \\
Both-edge Au-doped & 30.19 \\
\hline
\end{tabular}

\section{Charge density}

The distribution of difference charge density for Au-doping AGNRs with both-edge configurations are shown in Figure 2 (a). It shows that the charge density is transferred between the $\mathrm{Au}$ and $\mathrm{C}$ atoms and located mainly on the $\mathrm{Au}$ atoms. Charge densities of about $0.8895 \mathrm{e}^{-} / \AA^{3}$ is transferred from $\mathrm{C}$ atoms to Au atoms for both-edge doping are shown in Figure 2 (b). Furthermore, the orbital hybridization between the $\mathrm{Au}$ and $\mathrm{C}$ atoms is obvious and the bonding charge is 
completely located between the $\mathrm{Au}$ atoms, as shown in Figure 2 (a). So the stability and conductivity of the system undergo sizable changes due to the charge transfer.

(a)

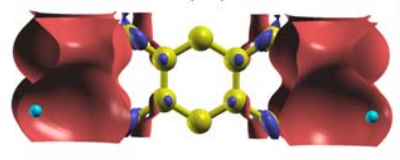

(b)

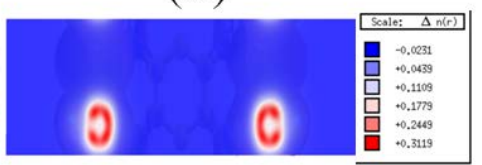

Fig.2. (a) The isosurface plots of the difference charge density for both-edge doped AGNRs, (b) quantity plots of the difference charge density for one-edge doped.

Isovalues: 0.005 and -0.005 a.u.

\section{Band structure}

The energy band structures for the Au-doping both-edge configurations is calculated as shown in Figure 3. The energy bands appear near the Fermi level due to additional $\pi^{*}$ electrons from the $\mathrm{Au}$ atoms. Thus the Fermi level crosses the conduction band and the ribbon becomes metallic. Near the Fermi level the valence band structure is disturbed due to Au doping. Figure 3 shows extra bands at near Fermi level in the valence band, which is cause to promote conductivity for AGNRs. Furthermore, we find that both-edge Au-doped ribbons are the most conductive, as they have more conducting channels than the other types of AGNRs. The GNRs can therefore provide a wide range of possible electronic properties based on the same ribbon structure with different doping configurations.

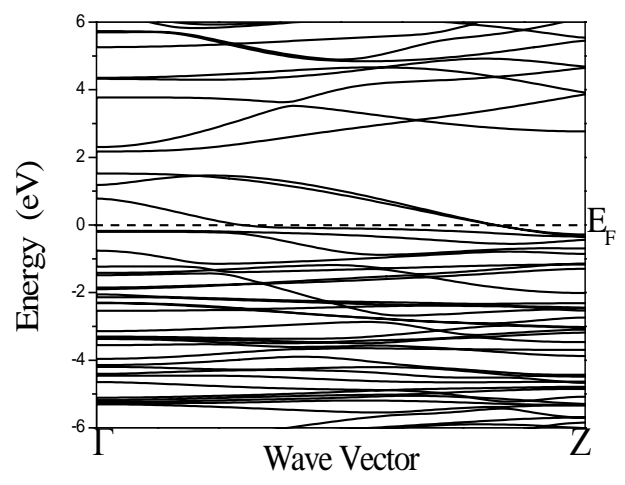

Fig.3. Schematic of band structures of doped both-edge AGNRs.

\section{Project density of states}

The valence band and conduction band of Au both-edge-doping AGNRs are mainly contributed by $\mathrm{C}-2 p$, Au- $6 s, 6 p, 5 d$ and $\mathrm{H}-1 s$ states, as shown as figure 4 . It shows the $\mathrm{C}-2 p$ and Au- $5 d$ electrons are strongly hybridized due to the similar form of the PDOS distributions in the valence band and conduction band, which causes the Fermi level turn into the valence band. The strong hybridization can be explained in terms of charge transfer between Au-6p and $\mathrm{H}-1 s$ atoms. The sharp peak in conduction band corresponds to electronic states of C-2p and $\mathrm{H}-1 s$. The sharp peaks in valance band of $\mathrm{Au}$ both-doped AGNRs at $-2.32 \mathrm{eV},-3.37 \mathrm{eV}$ and $-4.46 \mathrm{eV}$ come mainly from the Au-5d electronic states. A localization state is induced due to the absence of the bonding charge between $\mathrm{Au}$ and $\mathrm{H}$ atoms, which contributes to $\mathrm{H}-1$ s electronic states at $-0.21 \mathrm{eV}$ near the Fermi level. In addition, for the both edge Au-doped AGNRs, the PDOS analysis indicates C-2p, Au-6s, 6p, 5d, $\mathrm{H}-1$ s electrons mainly contribute to electronic states near the Fermi level. 


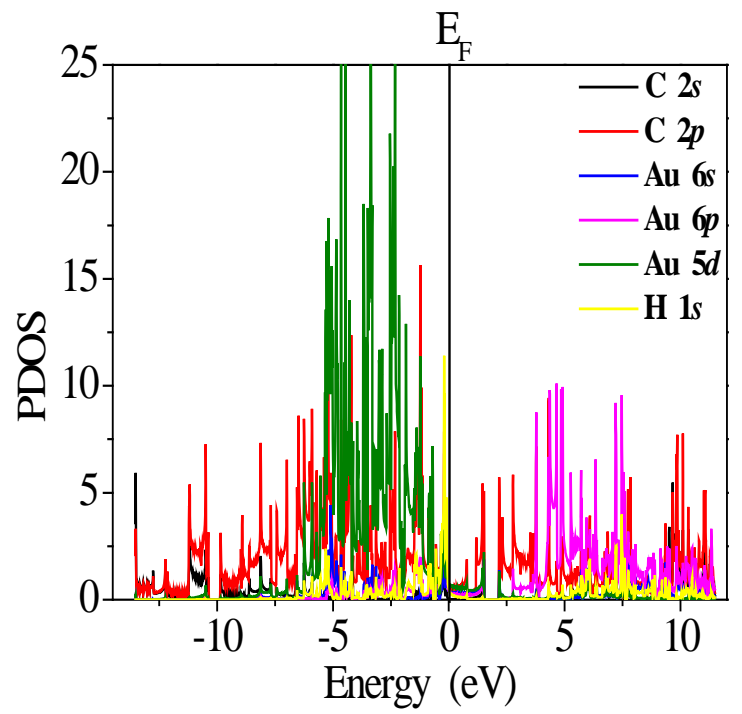

Fig.4. Projected density of states of AGNRs both-edge Au-doped.

\section{Conclusion}

The charge density, energy band structure and project density of states of both-edge Au-doped narrow AGNRs is calculated by the first principle based on DFT. Our results indicate that the bonding charge between the $\mathrm{C}$ and $\mathrm{Au}$ atoms is transferred and the difference charge density is mainly located on the Au atoms. The strong hybridization between the C- $2 p, \mathrm{Au}-5 d, 6 s, 6 p$ and $\mathrm{H}-1 s$ electrons in the valence band and conduction band, the Au doping induce the Fermi level turned into the conduction band, which become into metal. Therefore, the nature AGNRs is changed into degenerate semiconductor. It can be seen that Au both-edge doped AGNRs provide a wide range of possible electronic properties based on the same ribbon structure.

\section{Acknowledgments}

This work is supported by National Natural Science Foundation of China No. 11464034, Doctoral Scientific Research Foundation of Inner Mongolia University for Nationality No. BS319, National Natural Science Foundation of China No. 11304143 and Natural Science Foundation of Inner Mongolia No. 2013MS0807.

\section{References}

[1] M. Ezawa, Phys. Rev. B 73 (2006) 045432

[2] X.T. Jia, J. Campos-Delgado, M. Terrones, V. Meunier and M.S. Dresselhaus, Nanoscale 3 (2011) 86

[3] Q.M. Yan, B. Huang, J. Yu, F.W. Zheng, J. Wu, B.L. Gu, F. Liu, W.H. Duan, Nano Lett. 7 (2007) 1459

[4] B. Huang, Z.Y. Li, Z.R. Liu, G. Zhou, S.G. Hao, J. Wu, B.L. Gu, and W.H. Duan J. Phys. Chem. C 112 (2008) 13442-13446

[5] S.S. Chauhan, P. Srivastava, A.K. Shrivastava, Solid State Communication 154 (2013) 69-71

[6] S.S Yu, W.T. Zheng, Q.B. Wen, Q. Jiang, CARBON 46 (2008) 537-543

[7] A.Kokalj, Computer graphics and graphical user interfaces as tools in simulations of matter at the atomic scale, Comp. Mat. Sci. 28 (2003) 155

[8] M. Methfessel, A.T. Paxton, Phys. Rev. B 40 (1989) 3616

[9] Giannozzi P et al. 2009 J. Phys. Condens. Matter 21395502 